\title{
OPTIMIZATION OF 16-APSK BY MAXIMIZING MUTUAL INFORMATION CRITERIA IN SC-FDMA COMMUNICATION SYSTEMS
}

\author{
Denis DUPÁK, Juraj GAZDA, Dušan KOCUR, Mário PETRÍK \\ Department of Electronics and Multimedia Communications, Faculty of Electrical Engineering and Informatics, \\ Technical University of Košice, Letná 9, 04200 Košice, Slovak Republic, tel. +421 556024220 , \\ e-mail: denis.dupak@tuke.sk,juraj.gazda@tuke.sk,dusan.kocur@tuke.sk, mario.petrik@tuke.sk
}

\begin{abstract}
Single Carrier Frequency Division Multiplexing (SC-FDMA) scheme has been considered to be promising technology for the uplink of the $4 G$ mobile communication systems. The main disadvantage of SC-FDMA is the high envelope fluctuation of signal resulting in a presence of nonlinear distortion in transmitted signal due to the transmitter high power amplifier (HPA). In order to improve the SC-FDMA performance, the 16-ary Amplitude Phase Shift Keying (16-APSK) is proposed as a base-band modulation scheme for SC-FDMA instead of conventional M-QAM. The different types of 16-APSK modulations will be investigated and positions of constellation points will be optimized according to maximizing Mutual Information (MI) criteria. For given value of SNR will be found optimal 16-APSK modulation and it will be proposed adaptive approach of 16-APSK modulation. The performance properties of adaptive approach of 16-APSK and 16-QAM based SC-FDMA transmission scheme will be illustrated using numerical results. The obtained results will show that optimized 16-APSK modulation in SC-FDMA transmitted system provide better performance than 16-QAM SC-FDMA system if the nonlinear model of HPA has to be assumed.
\end{abstract}

Keywords: SC-FDMA, APSK, HPA, Mutual Information, Optimization

\section{INTRODUCTION}

The demand for high data rates transmission over multipath radio channels has increased rapidly. To fulfill this requirement, utilization of multi-carrier based transmission techniques seems to be an inevitable solution. Very promising modulation technique, which satisfy communication system requirements is Orthogonal Frequency Division Multiplexing (OFDM). The OFDM is spectrally efficient modulation technique that has recently gained much popularity due to high speed and very effective data transmission over multipath fading channels. The OFDM based transmission systems are characterized by large number of benefits in comparison with traditional schemes. This makes it superior compared to previously introduced wireless standards [1].

On the other hand, OFDM has some well known drawbacks. To perform interference free demodulation the subcarriers have to remain orthogonal, otherwise system performance will degrade. Therefore, OFDM is very sensitive, to frequency offset originating from the mismatch of transmitter and receiver local oscillators. The time domain OFDM signal is a sum of a large number of complex sinusoids, which means that, according to the central limit theorem, the amplitude distribution will be Gaussian, leading to a large peak to average power ratio (PAPR) of the signal. Hence, a power amplifier with a relatively large linear range is required otherwise nonlinear effects will severely degrade the system performance [2].

Large sensitivity to nonlinear amplification has greatly limited the practical applications of OFDM transmission systems. In order to alleviate the effect of nonlinear amplification in OFDM transmission systems, many approaches based on different techniques have been introduced. Frequently used solution in the transmitter is to back-off the operating point of nonlinear amplifier, but this approach results in significant power efficiency penalty. Alternative approaches for OFDM performance improvement are realized by applications of other usually computational demanded PAPR reduction methods at the transmitter side, for example pre-coding of signals (SCFDMA), active constellation extension [3], tone reservation [4] or selected mapping [5]. Another well known and promising solutions to reduce influence of nonlinear distortion are the application of nonlinear iterative detection [6] at the receiver side.

In the core part of this paper, based on results of the presented analysis, we propose to use the baseband signal constellation optimization to further reduce the signal envelope fluctuation of SC-FDMA scheme, which was introduced in Long Term Evolution (LTE) uplink. SCFDMA is characterized by significant lower PAPR compared to that of OFDM, nevertheless, it is still higher compared to the conventional single carrier systems. It should be noted that by observing ongoing research, nonlinear amplification in the LTE uplink still attracts the attention and remains the key design problem to be solved here.

In this paper, we propose to use the optimized 16APSK baseband signal modulation to further reduce the signal envelope fluctuation instead of conventional originally considered M-QAM modulation scheme. Basically, the idea presented in this paper is inspired by the recent progress in the satellite communications. DVBS2 represents the new standard, which is designed as a successor for the popular DVB-S digital television broadcast standard [7]. The recent technical enhancements in satellite broadcasting require the exploitation of highly efficient power and spectrally modulation schemes designed to operate over the nonlinear satellite channel environment. In this regard, M-APSK represents an attractive modulation scheme for digital transmission over nonlinear satellite channels.

Optimization of 16-APSK constellation was made by using maximizing Mutual Information (MI) criteria, which 
ensures that the maximum amount of information par symbol is transmitted over communication channel. The aim of this paper is to find optimal 16-APSK for different scenarios and study the performance of optimal 16-APSK modulations in SC-FDMA transmission systems. It was investigated 16-APSK constellations with various number of constellation points on each ring $(4+12$ APSK, 5+11 APSK, 6+10 APSK). Also, it was investigated the impact of distance between the rings and rotation of rings. Obtained results are compared to 16-QAM modulation which is commonly used by LTE.

The rest of the paper is organized as follows. In the next section, the analysis of the SC-FDMA signal is presented. The definition of M-APSK modulation is in Section 3 and the maximizing MI criteria is discussed in Section 4. Section 5 is devoted to the computer simulations confirming the feasibility of 16-APSK application. Finally, conclusions and final remarks to this contribution are drawn in Section 6.

\section{SYSTEM MODEL}

In SC-FDMA, a block of $N$ data symbols from some baseband modulation alphabet, such as QPSK, M-QAM or M-APSK is first applied to a size- $N$ discrete Fourier Transform (DFT). The output of the DFT is then applied to consecutive inputs of a size- $M$ inverse DFT (IDFT), where $M \leq N$, and the unused inputs of the IDFT are set to zero. Finally, as in OFDM a cyclic prefix is inserted to each transmitted block.

Let $a_{i}, i=0 \ldots N-1$ be the complex data symbols, then the signal at the output of DFT precoder can be expressed as [6]:

$S_{k}=\frac{1}{\sqrt{N}} \sum_{k=0}^{N-1} a_{i} e^{-j 2 \pi i k / N}, k=0, \ldots, N-1$

In the OFDM block the $N$ pre-coded data symbols are transmitted over $N$ consecutive subcarriers. Consider a baseband OFDM symbol $s(t)$ defined over the time interval $t \in\left[0 ; T_{s}\right]$

$$
s(t)=\frac{1}{\sqrt{N}} \sum_{k=0}^{N-1} S_{k} e^{j 2 \pi\left(k+k_{0}\right) t / T_{S}},
$$

where $k_{0}$ is the position of the first assigned subcarrier. For the sake of brevity and without loss of generality we assume $k_{0}=0$. If $\mathrm{s}(\mathrm{t})$ is sampled at a frequency $L N=T_{s}$, where $L=M / N$ is the oversampling factor and $N=T_{s}$ is the Nyquist rate, the signal at the output of the SC-FDMA modulator is

$$
S_{n}=\frac{1}{\sqrt{N}} \sum_{k=0}^{N-1} S_{k} e^{j 2 \pi k n / M}, n=0, \ldots, M-1
$$

The expression above can be computed by means of a length- $M$ scaled IDFT. Therefore, from (1) and (3) the SC-FDMA signal can be expressed as

$$
\begin{aligned}
& S_{n}=\frac{1}{\sqrt{N}} \sum_{k=0}^{N-1}\left(\frac{1}{\sqrt{N}} \sum_{i=0}^{N-1} d_{i} e^{-j 2 \pi i k / N} e^{j \pi i}\right) e^{j 2 \pi k n / M} \\
& =\frac{1}{N} \sum_{i=0}^{N-1}(-1)^{i} d_{i} \sum_{k=0}^{N-1} e^{j 2 \pi k(n-L i) / M} \\
& =\frac{1}{N} \sum_{i=0}^{N-1} d_{i}^{\prime} \sum_{k=0}^{N-1} e^{j 2 \pi k(n-L i) / M}
\end{aligned}
$$

Note that for the mapping schemes used in LTE uplink $(-1)^{i} d_{i}$ is always a point of the constellation, therefore we denote it as $d_{i}^{\prime}$.

Now, let us analyze the SC-FDMA signal at sample position multiple of the spreading factor. If $n=L r$, the time domain signal in (4) reduces to

$S_{L r}=\frac{1}{N} \sum_{i=0}^{N-1} a_{i} \sum_{i=0}^{N-1} e^{2 \pi k(r-1) / \mathrm{M}}$

$=\frac{1}{N}(a_{r} \underbrace{\sum_{k=0}^{N-1} e^{2 \pi k_{0} / N}}_{N}+\sum_{i=0, i \neq r}^{N-1} a_{i} \underbrace{\sum_{k=0}^{N-1} e^{2 \pi k(r-i) / \mathrm{M}}}_{0})$

$=a_{r}$

which means that the $L r$-th sample of the time domain SCFDMA signal is equal to the data symbol $a_{r}$. The samples at positions $n \neq L r$ describe the transition of the timedomain signal between the values $a_{r}$ and $a_{r+l}$.

However, between those samples the signal fluctuates. To understand why this happens, we must consider the way oversampling is implemented. In SC-FDMA oversampling is done by zero padding the complex precoded baseband signal before applying IFFT. Notice that this means that no discrete frequency outside the transmission bandwidth is allowed. Therefore, if an abrupt change in the value of contiguous data symbols occurs, since a fast transition in the time domain samples is not possible because of the spectral properties imposed by frequency-domain oversampling, it can only be solved by showing a smooth transition at expenses of the peak values. This is similar to the effect observed when a rectangular pulse is low-pass filtered. On the other hand, if we are allowed to perform some spectral outgrowth we will be able to reduce the fluctuations of the transmitted signal since faster transitions will be possible.

Major conclusion from the presented analysis is that while PAPR of the OFDM signal is regardless of the mapping scheme being used, in SC-FDMA scenario the PAPR might be significantly reduced by the careful selection of the baseband modulation scheme. In other words, using the baseband modulation scheme with reduced ring constellation size (i.e. number of different normalized energy levels) might improve the system performance in the nonlinear environment thanks to the inherent reduced PAPR. Further improvement of system performance can be achieved by using suitable version of 16-APSK for each investigated scenatio. Analisis of different 16-APSK modulations and applied optimization will be discussed in next two sections. 


\section{APSK MODULATION SCHEME DEFINITION}

M-APSK constellations are composed of $n_{r}$ concentric rings. Each ring of constellation diagrams uniformly spaced phase shift points. The signal constellation points are complex numbers, drawn from the set [7]:

$$
\mathcal{X}=\left\{\begin{array}{lc}
\hline r_{1} \exp \left[j\left(\left(2 \pi / n_{1}\right) i+\theta_{1}\right)\right] & i=0, \ldots, n_{1}-1 \\
r_{2} \exp \left[j\left(\left(2 \pi / n_{2}\right) i+\theta_{2}\right)\right] & i=0, \ldots, n_{2}-1 \\
\vdots & \vdots \\
r_{N_{R}} \exp \left[j\left(\left(2 \pi / n_{R}\right) i+\theta_{N_{R}}\right)\right] & i=0, \ldots, n_{N_{R}}-1 \\
\hline
\end{array}\right.
$$

where $n_{l}, r_{l}$ and $\theta_{l}$ denote the number of constellation points, the radius and relative phase shift, respectively of $l$-th ring. In the case of the optimum 16-APSK, its parameters $n_{l}, r_{l}$ and $\theta_{l}$ are designed using a suitable optimization criterion. In the case of the optimum 16APSK, its parameters $n_{l}, r_{l}$ and $\theta_{l}$ are designed using a suitable optimization criterion. Here we have selected criteria, which is based of maximizing MI.

Typical example of the different 16-APSK signal constellations and 16-QAM constellation is given in Fig. 1. The notable difference between these particular constellation schemes is that while in 16-QAM the constellation symbols occupy three constellation rings (i.e. $R=3$ ), in the latter example for 16-APSK, $R=2$.
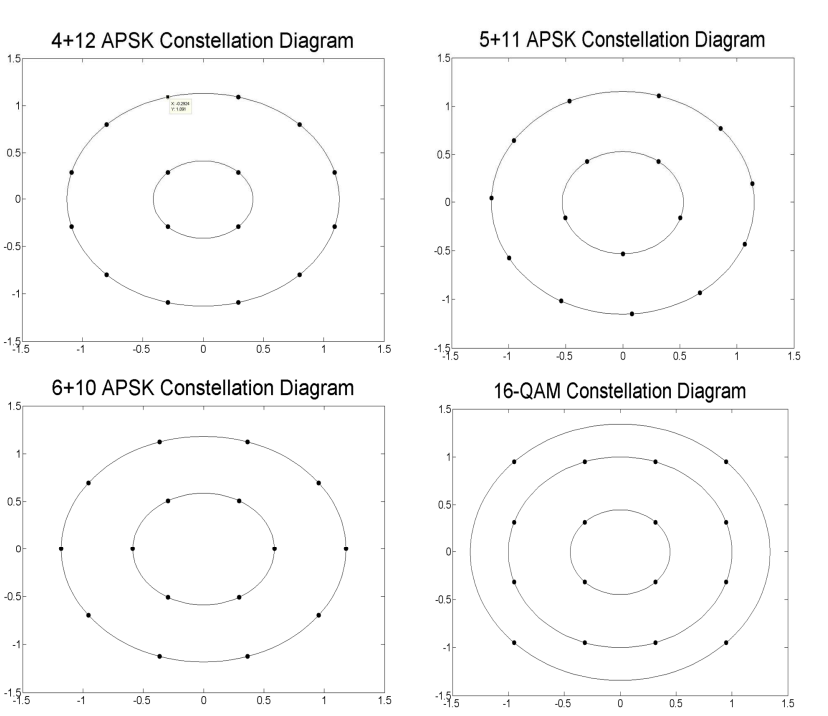

Fig. 1 Figure caption Constellation diagrams of 4+12 APSK, 5+11 APSK, 6+10 APSK and 16-QAM

Taking into account (4) and the particular characteristics of 16-QAM(16-APSK) signal constellations, one would expect that the PAPR characteristic of 16-APSK SC-FDMA signal might be potentially lower than that of 16-QAM SC-FDMA. This issue was investigated in our previous research, where the issue of the envelope fluctuation reduction of SC-FDMA signal by using baseband modulation scheme with lower number of energy levels was explained in more detail [8]. We can see from mentioned article, that M-APSK modulation scheme provide better system performance than M-QAM for SC-FDMA transmission system in highly nonlinearly disturbed scenarios. This feature of MAPSK modulation is combined with maximizing of distance between the constellation points, which reduce the effect of Additive white Gaussian noise (AWGN) interference for low signal-to-noise ratio (SNR).

In this article, we will focus to investigate three different types $(4+12,5+11,6+10)$ of 16-APSK modulations schemes, which are illustrated in Fig.1. These variants of 16-APSK modulation schemes were optimized according maximizing MI criteria, which was described in next section.

\section{MAXIMAZING MI OPTIMIZATION CITERIUM}

The optimization of APSK constellation scheme is carried out using the conventional criterion of maximum MI transmitted over communication channel. This issue was solved via brute-force approach, where all possible options were investigated. For a fixed relative phase shift $\theta_{l}$ of the $l$-th ring, the constellation optimization has to obey the following basic constraints:

$N_{1}+N_{2}=M=16$

$\rho=r_{2} / r_{1}>1, \quad r_{1}=1$,

$\frac{N_{1} r_{1}^{2}+N_{2} r_{2}^{2}}{16}=1$,

where (9) is the constant-energy condition.

The average mutual information (assuming equiprobable symbols) for a given signal set $\mathrm{X}$ provides the maximum transmission rate at which error-free transmission is possible with such signal set, and is given by $[8]$ :

$$
\begin{aligned}
& f(\chi)=C=\log _{2} M- \\
& E_{x, \mathrm{n}}\left\{\log _{2}\left[\sum_{x^{\prime} \in \chi} \exp \left(-\frac{1}{N_{0}}\left|\sqrt{E_{S}}\left(x-x^{\prime}\right)+n\right|^{2}-|n|^{2}\right)\right]\right\} .
\end{aligned}
$$

Interestingly, for a given signal-to-noise ratio, or equivalently, for a given spectral efficiency $\eta_{s}$, an optimum constellation can be obtained.

The Fig.2 illustrates overview of MI of 16-APSK modulation for linear model of HPA. It was used three different types of 16-APSK modulation (4+12 APSK, 5+11 APSK, 6+10 APSK). MI for those constellation diagrams was computed for values of $r_{2} / r_{1}$ from interval 1.25 up to 5 . We used increment step equals 0.125 and $\mathrm{MI}$ was computed for values of SNR from interval 8 up to 20 with increment step 2 . Not all calculated data was plotted because of clarity of figure. As we can see from the Fig.2, $6+10$ APSK modulation gives the maximal values of MI for all investigated SNR and $r_{2} / r_{1}$ for SNR in interval from $8 \mathrm{~dB}$ up to $14 \mathrm{~dB}$ and for the interval from $14 \mathrm{~dB}$ up to $20 \mathrm{~dB}$ the best results are provided by $5+11$ APSK. 


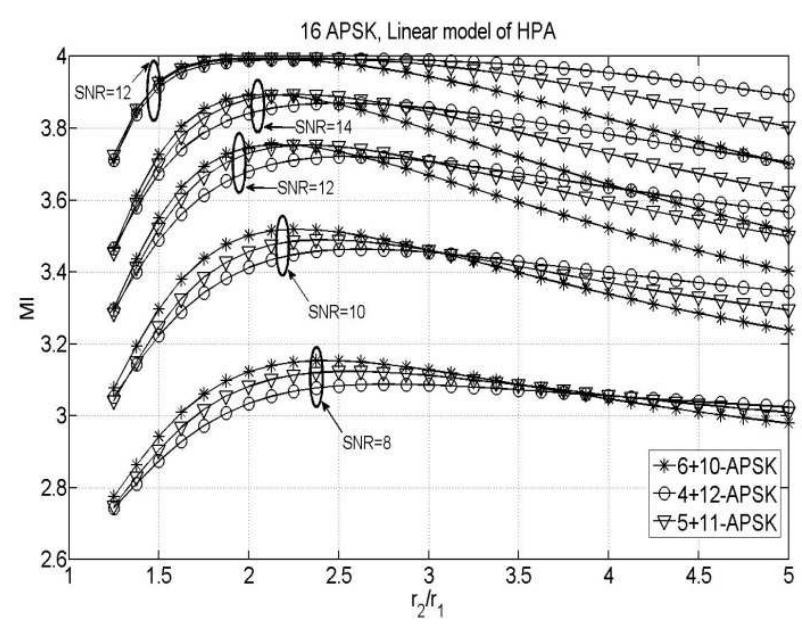

Fig. 2 MI of 4+12 APSK, 5+11 APSK, 6+10 APSK for linear model of HPA

The Fig.3 illustrates overview of MI of 16-APSK modulation for nonlinear model of HPA. It was used saleh model of HPA with Input Back Off parameter (IBO) equals 0 . MI for those constellation diagrams was computed for values of $\mathrm{r}_{2} / \mathrm{r}_{1}$ from interval 1.5 up to 10 . We used increment step equals 0.125 and MI was computed for values of SNR from interval 8 up to 20 with increment step 2. As it was in linear scenario, not all calculated data was plotted because of clarity of figure. From Fig. 3 we can observe that maximal MI can be reached by using $6+10$ APSK modulation for SNR from interval 8dB up to $14 \mathrm{~dB}$ and $5+11$ APSK modulation in interval from $14 \mathrm{~dB}$ up to $20 \mathrm{~dB}$.

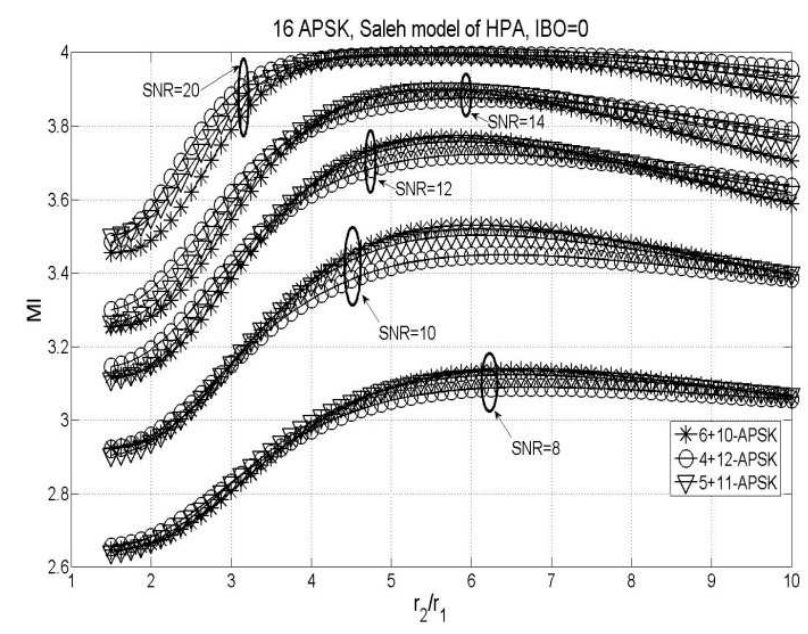

Fig. 3 MI of 4+12 APSK, 5+11 APSK, 6+10 APSK for Saleh model of HPA, IBO $=0$

Exact values of $r_{2} / r_{1}$ with maximal MI for all three types of investigated 16-APSK modulations and related maximal MI are shown in Table 1 for linear scenario and in Table 2 for nonlinear scenario. Optimal 16-APSK modulations for linear scenario are highlighted with bold font.
Table 1 Optimal values of $r_{2} / r_{1}$ and related MI for linear model of HPA

\begin{tabular}{|l|l|l|l|l|l|l|l|}
\hline \multicolumn{7}{|c|}{ r/r1 } \\
\hline SNR & 8 & 10 & 12 & 14 & 16 & 18 & 20 \\
\hline $4+12$ & 2,75 & 2,625 & 2,625 & 2,625 & 2,5 & 2,375 & 2,375 \\
\hline $5+11$ & 2,5 & 2,375 & 2,375 & $\mathbf{2 , 2 5}$ & $\mathbf{2 , 2 5}$ & $\mathbf{2 , 1 2 5}$ & $\mathbf{2 , 2 5}$ \\
\hline $6+10$ & $\mathbf{2 , 3 7 5}$ & $\mathbf{2 , 2 5}$ & $\mathbf{2 , 1 2 5}$ & $\mathbf{2 , 1 2 5}$ & 2 & 2 & 2 \\
\hline \multicolumn{7}{|c|}{ MI } \\
\hline SNR & 8 & 10 & 12 & 14 & 16 & 18 & 20 \\
\hline $4+12$ & 3,008 & 3,464 & 3,721 & 3,869 & 3,95 & 3,981 & 3,993 \\
\hline $5+11$ & 3,124 & 3,49 & 3,753 & 3,894 & 3,962 & 3,987 & 3,996 \\
\hline $6+10$ & 3,154 & 3,52 & 3,755 & 3,894 & 3,959 & 3,986 & 3,995 \\
\hline
\end{tabular}

Table 2 Optimal values of $r_{2} / r_{1}$ and related MI for saleh model of $\mathrm{HPA}, \mathrm{IBO}=0$

\begin{tabular}{|c|c|c|c|c|c|c|c|}
\hline \multicolumn{7}{|c|}{ r/r1 } \\
\hline SNR & 8 & 10 & 12 & 14 & 16 & 18 & 20 \\
\hline $4+12$ & 6,75 & 6,375 & 6,125 & 6 & 5,875 & 5,625 & 5,625 \\
\hline $5+11$ & 6,375 & 6 & 5,875 & $\mathbf{5 , 5}$ & $\mathbf{5 , 5}$ & $\mathbf{5 , 5}$ & $\mathbf{5 , 2 5}$ \\
\hline $6+10$ & $\mathbf{6 , 3 7 5}$ & $\mathbf{6}$ & $\mathbf{5 , 6 2 5}$ & $\mathbf{5 , 5}$ & $\mathbf{5 , 2 5}$ & 5,25 & 5,125 \\
\hline \multicolumn{7}{|c|}{ MI } \\
\hline SNR & 8 & 10 & 12 & 14 & 16 & 18 & 20 \\
\hline $4+12$ & 3,09 & 3,45 & 3,723 & 3,876 & 3,948 & 3,98 & 3,993 \\
\hline $5+11$ & 3,123 & 3,503 & 3,757 & 3,899 & 3,963 & 3,987 & 3,996 \\
\hline $6+10$ & 3,165 & 3,531 & 3,769 & 3,899 & 3,963 & 3,986 & 3,995 \\
\hline
\end{tabular}

The obtained results were compared to conventional 16-QAM modulation and overview of MI preferences for linear and nonlinear scenarios was illustrated in Fig.4 and Fig.5. As it can be seen from Fig.4, MI of all investigated 16-APSK is almost the same as MI for 16-QAM. $6+10$ APSK provides slightly better performance than other cases. The breakthought in our investigation occurred if the nonlinear model of HPA was used. This case is illustrated in Fig.5. As we can see form the Fig.5, MI for 16-QAM modulation is considerably lower than 16APSK. The best performance is provided by $6+10$ APSK for SNR $8 \mathrm{~dB}$ up to $16 \mathrm{~dB}$ and 5+11 APSK for SNR from interval $14 \mathrm{~dB}$ up to $20 \mathrm{~dB}$. MI is the same for SNR equals $14 \mathrm{~dB}$ and $16 \mathrm{~dB}$. We concluded that for those values of SNR there are two optimal 16-APSK modulations and it is up to us which one we will choose in further investigation. Also we can choose one of those optimal modulations according some other criteria for example BER or MED (Minimum Euclid Distance).

For optimal 16-APSK modulation, which are shown in Table 1 and Table 2 were computed BER preferences in SC-FDMA communication system. For cases where two optimal 16-APSK modulations exist, the BER preferences were computed for both constellations and results were almost the same. 


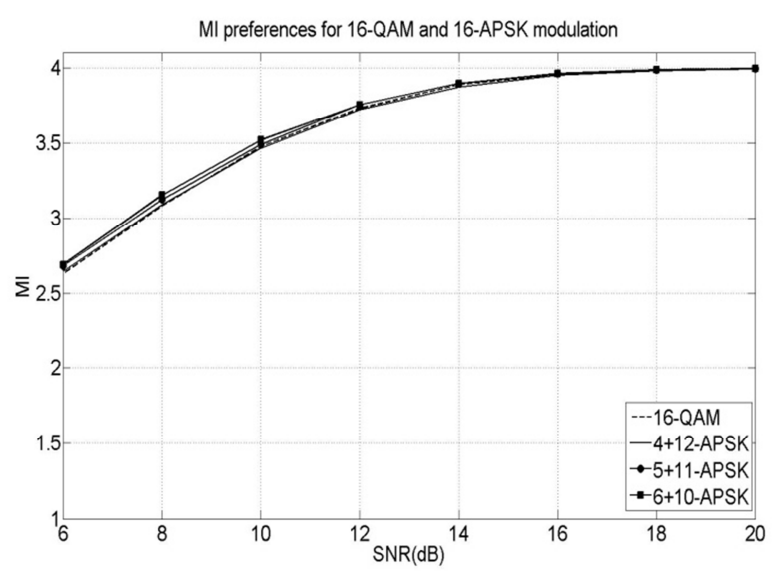

Fig. 4 MI comparison of 16-APSK and 16-QAM modulation for linear model of HPA

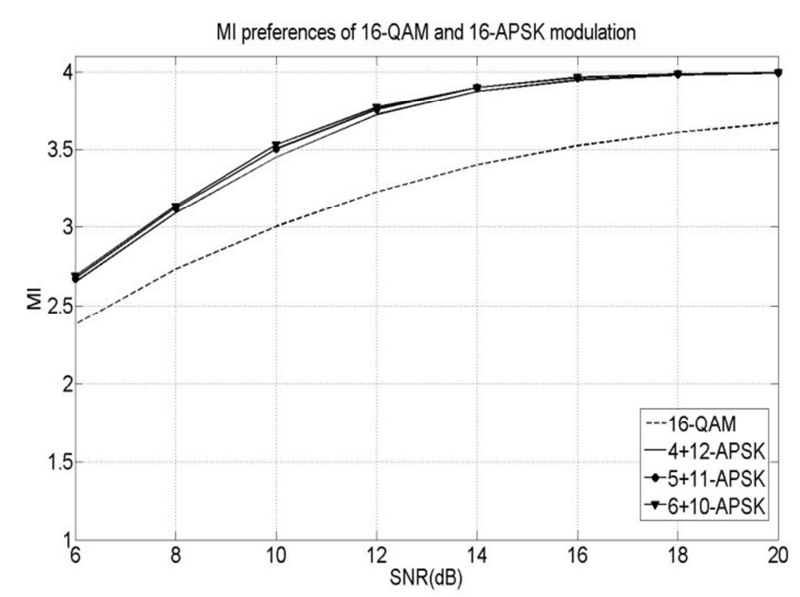

Fig. 5 MI comparison of 16-APSK and 16-QAM modulation for saleh model of HPA, IBO $=0$

In Fig.6 we can see comparison of BER preferences for 16-QAM modulation and 16-APSK modulation. For this scenario was used linear model of HPA and we can see from the figure that 16-QAM provides slightly better BER performance than optimal 16-APSK modulation.

Fig.7 illustrates BER performance of 16-QAM and optimal 16-APSK modulation for nonlinear model of HPA. It was used Saleh model of HPA with IBO parameter equals 0 . Optimal 16-APSK provides slightly better BER performance for nonlinear scenario for all investigated SNR values.

\section{CONCLUSIONS}

In this paper, we evaluated the performance of different variants 16-APSK baseband modulation for SCFDMA. 16-APSK modulations was optimized according to maximize MI criteria. It can be concluded that adaptive optimizing approach, when we choose the optimal 16APSK constellation for given SNR, provides high robustness against nonlinear amplification and its

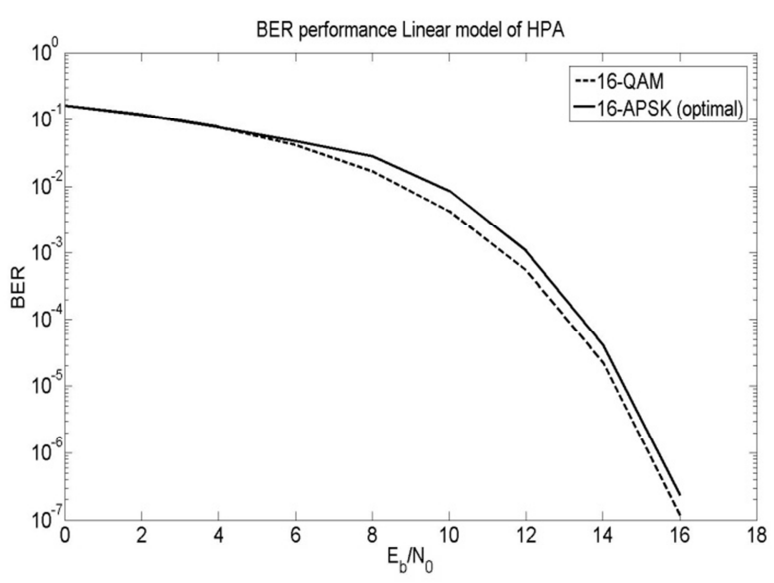

Fig. 6 BER comparison of 16-APSK and 16-QAM modulation for linear model of HPA

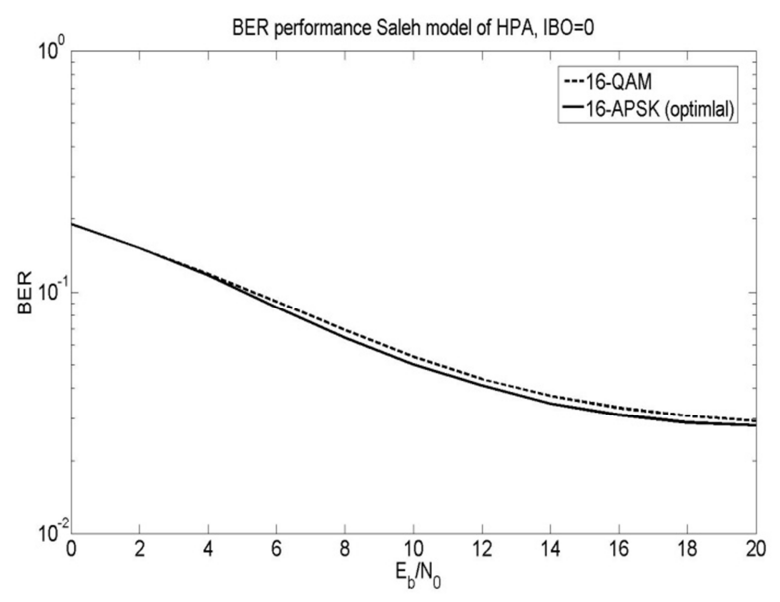

Fig. 7 BER comparison of 16-APSK and 16-QAM modulation for saleh model of $\mathrm{HPA}$, IBO $=0$

application is especially reasonable for highly nonlinearly disturbed scenarios. The obtained results show very clearly, that if the user employs optimal 16-APSK with required values of $r_{2} / r_{1}$, it will able to maximize transfer rate over the transmission channel especially for nonlinear scenario where BER performance was slightly better than 16-QAM modulation and it was used the 16-APSK modulation with better MI preferences than 16-QAM modulation. The adaptive optimal 16-APSK modulation technique might be further used together with some other PAPR reduction techniques and coding techniques to provide better transmission preferences for SC-FDMA communication systems.

\section{ACKNOWLEDGMENTS}

This work is the result of the project implementation Short-Range UWB Sensor Networks for Detection, Localization and Tracking of Moving Persons (UWBSeNet) (project VEGA 1/0563/13). 


\section{REFERENCES}

[1] DAHLMAN, E. - PARKVALL, S.: $3 G$ Evolution, HSPA and LTE for Mobile Broadband, Academic Press, Oxford UK (2007).

[2] MYUnG, H. G. - GOODMAN, D. J.: A New Air Interface for Long Term Evolution, Winchester, UK: Wiley (2008).

[3] IKRONGOLD, B. S. - JONES, D. L.: PAR Reduction in OFDM via Active Constellation Extension, IEEE Transactions on Broadcasting. vol. 49. no. 3, pp. 258-268, Sep. 2003.

[4] DUEMAL, M. - BEHRAVAN, A. - ERIKSSON, T. - PIJOAN, J. L.: Evaluation of performance improvement capabilities of PAPR reducting methods, Wireless Personal Communications, vol. 47, No. 1, pp. 137-147, Oct. (2008).

[5] CIMINI, L. J. - SOLLENBERGER, N. R.: Peak-toAverage Power Ratio Reduction of an OFDM SignalUsing Partial Transmit Sequences, IEEE Communications Letters, vol. 4, No. 3, pp. 86-88, Mar. (2000).

[6] GAZDA, J.: Multicarrier based transmission system undergoing nonlinear amplification, Doctoral Thesis, Košice: Technical University of Košice, Faculty of Electrical Engineering and Informatics, (2010).

[7] GAUDENZI, R. - FABREGAS, A. G. MARTINEZ, A.: Turbo-coded APSK modulations design for satellite broadband communications, Int. J. Satell. Commun. Network. (2006).

[8] GAZDA, J. - DUPÁK, D. - KOCUR, D.: M-APSK Modulation for SC-FDMA Communication Systems, In: Radioelektronika 2011 : proceedings of 21th international conference : April 19-20, 2011, Brno, Czech Republic. - Brno : Brno University of Technology, 129-132 (2011).

Received February 24, 2014, accepted March 18, 2014

\section{BIOGRAPHIES}

Denis DUPÁK was born in 1984 in Kosice (Slovakia). He received his M.Sc. degree in Electronics and Telecommunications in 2008 from the Faculty of Electrical Engineering and Informatics, Technical University of Kosice and currently he is working towards his $\mathrm{PhD}$. at the same university. His research interests include effects of non-linear amplification on multicarrier transmission schemes and design of baseband modulations schemes in SC-FDMA transmission systems.

Juraj GAZDA was born in 1984 in Kosice (Slovakia). He received his M.Sc. degree in Electronics and Telecommunications in 2007 from the Faculty of Electrical Engineering and Informatics, Technical University of Kosice and he recieved $\mathrm{PhD}$. degree at the same university. During 2006/2007 he spent one semester at Delf University of Technology, The Netherlands. Since 2009, he is with Research group in Electromagnetism and Communications, La Salle, Universitat Ramon Llull, Barcelona, Spain as a guest researcher working in the area of nonlinear effects in OFDM. His research interests include effects of non-linear amplification on multicarrier transmission schemes and design of advanced receivers for Beyond $3 \mathrm{G}$ and $4 \mathrm{G}$ transmission systems.

Dušan KOCUR was born in 1961 in Košice, Slovakia. $\mathrm{He}$ received the Ing $(\mathrm{MSc})$ and $\mathrm{CSc}(\mathrm{PhD})$ in radioelectronics from the Faculty of Electrical Engineering, Technical University of Košice, in 1985 and 1990. He is full professor at the Department of Electronics and Multimedia Communications of his Alma Mater. His research interests are digital signal processing, spreadspectrum communication systems (MC-CDMA, CDMA), UWB technology and e-learning.

Mário PETRÍK was born in 1988 in Kosice (Slovakia). He is student of Faculty of Electrical Engineering and Informatics in Technical University of Kosice and currently he is working towards his M.Sc. at the same university. His research interests include effects of nonlinear amplification on multicarrier transmission schemes. 